\title{
Potencialidad de los espacios públicos abiertos para las relaciones intergeneracionales. Un estudio de caso en la ciudad de Santa Cruz de Tenerife (Canarias, España)
}

\author{
Carmen-Rosa Delgado-Acosta \\ Carmen-Gloria Calero-Martín \\ Herminia González-Bencomo \\ Universidad de La Laguna. Departamento de Geografía e Historia \\ cdelgado@ull.edu.es \\ cgcalero@ull.edu.es \\ gonzalez.bencomo.22@ull.edu.es
}

\section{Resumen}

El acelerado proceso de envejecimiento de las sociedades avanzadas ha suscitado la preocupación por fomentar la interacción de los mayores con los jóvenes para conseguir sociedades más cohesionadas. Los espacios públicos abiertos, al ser los lugares de concurrencia de usuarios de diferentes edades, son los escenarios idóneos para lograr la intergeneracionalidad. Para que esta sea posible es indispensable que se lleven a cabo actividades de encuentro, ya sean inducidas a partir de programas intergeneracionales o surjan de forma espontánea porque el diseño del espacio público lo estimula. Mediante las metodologías cuantitativa y cualitativa y con el objeto de constatar las relaciones intergeneracionales espontáneas en los espacios públicos abiertos y la potencialidad de los mismos para su desarrollo, se analiza el uso y la apropiación de jóvenes y mayores en dos plazas de Santa Cruz de Tenerife y los factores que pueden contribuir a que los espacios públicos resulten lugares para la interrelación generacional.

Palabras clave: espacio público; programas intergeneracionales; personas jóvenes; personas mayores; Santa Cruz de Tenerife. 
Resum. Potencialitat dels espais públics oberts per a les relacions intergeneracionals. Un estudi de cas a la ciutat de Santa Cruz de Tenerife (Canàries, Espanya)

L'accelerat procés d'envelliment de les societats avançades ha suscitat la preocupació per fomentar la interacció de les persones grans amb les persones joves per tal d'aconseguir societats més cohesionades. Els espais públics oberts, com que són els llocs de trobada dels usuaris de diferents edats, esdevenen els escenaris idonis per assolir la intergeneracionalitat. Per fer-la possible és indispensable que s'hi desenvolupin activitats de trobada, bé siguin induïdes a partir de programes intergeneracionals o bé apareguin de manera espontània perquè el disseny de l'espai públic així ho estimuli. Mitjançant les metodologies quantitativa i qualitativa i amb l'objectiu de constatar les relacions intergeneracionals espontànies en els espais públics oberts i la potencialitat dels mateixos per al seu desenvolupament, s'analitza l'ús i l'apropiació de les persones joves i grans en dues places de Santa Cruz de Tenerife i els factors que poden contribuir que els espais públics resultin llocs per a la interrelació generacional.

Paraules clau: espai públic; programes intergeneracionals; persones joves; persones grans; Santa Cruz de Tenerife.

Résumé. Potentiel des espaces publics ouverts pour les relations intergénérationnelles. Étude de cas dans la ville de Santa Cruz de Tenerife (îles Canaries, Espagne)

Le processus accéléré de vieillissement des sociétés avancées suscite l'intérêt pour encourager l'interaction des personnes âgées avec les jeunes afin d'obtenir des sociétés plus cohésives. Les espaces publics ouverts, en tant que lieux de rencontre d'usagers d'âges différents, sont les lieux idéaux pour atteindre l'intergénération. Dans ce but, il est indispensable que soient menées des activités de rencontre, qu'elles soient induites par des programmes intergénérationnels ou qu'elles surgissent de manière spontanée, stimulées par la conception même de l'espace public. Au travers de méthodologies quantitative et qualitative, et dans le but de constater les relations intergénérationnelles spontanées dans les espaces publics et leur potentiel pour leur développement, l'usage et l'appropriation des jeunes et des personnes âgées dans deux places de Santa Cruz de Tenerife sont analysés, ainsi que les facteurs qui pourraient contribuer à ce que les espaces publics soient finalement des lieux pour l'interaction générationnelle.

Mots-clés: espace public; programmes intergénérationnels; personnes jeunes; personnes âgées; Santa Cruz de Tenerife.

Abstract. The potential of open public spaces for intergenerational relationships: A case study in the city of Santa Cruz de Tenerife of the Canary Islands, Spain

A common concern in ageing societies has been to promote interaction between old and young people as a measure to achieve social cohesion. Open public spaces work as places where different age groups coexist, thus they could serve as scenarios to achieve intergenerationality. To that end, it is necessary to enact an agenda for meeting activities, whether planned - through intergenerational programs - or spontaneous, but taking advantage of the design of public spaces. The aim of this work is to explore casual, unplanned intergenerational relationships in open public spaces, and the potential for developing them. Based on quantitative and qualitative methods, this paper analyzes young and old peoples' appropriation of two squares in Santa Cruz de Tenerife, as well as the factors that guarantee intergenerational relationships in public spaces.

Keywords: public space; intergenerational programs; young people; old people; Santa Cruz de Tenerife. 


\section{Sumario}

\section{Introducción 4. Las relaciones intergeneracionales \\ 2. Objetivos y metodología \\ en el espacio público}

3. Los espacios públicos de observación: la Alameda del Duque de Santa Elena y la plaza Weyler
5. Programas intergeneracionales y espacio público abierto

6. Conclusiones

Referencias bibliográficas

\section{Introducción}

Los espacios públicos abiertos son lugares de encuentro y de interacción social utilizados por personas diferentes y de distintas generaciones. Dentro del conjunto de usuarios, jóvenes y mayores son dos colectivos que comparten con mucha frecuencia parques, plazas y paseos. La posibilidad de que en estos lugares se puedan consolidar relaciones entre ambos grupos de edad supone una gran oportunidad para las sociedades envejecidas, en las que se necesita cohesionar a las distintas generaciones para conseguir una «sociedad para todas las edades» (ONU, 2003), más solidaria e inclusiva ${ }^{1}$.

Sin embargo, en las sociedades occidentales, las relaciones entre jóvenes y mayores fuera del entorno familiar no son fáciles de establecer, pues las percepciones y las actuaciones de ambos grupos suelen ser distintas. Además, dichas relaciones entre personas desconocidas no solo son complicadas sino que, además, no son uniformes; muy al contrario, son diversas según los ambientes y los distintos contextos culturales y constituyen un aspecto más de la identidad social, que es muy compleja, e incluye variables como la cultura, la etnia, la clase social, el género, la religión, etc.; en definitiva, depende de los contextos y de las experiencias de cada individuo.

El proceso de envejecimiento que afecta a las poblaciones actuales convierte, no obstante, las relaciones entre jóvenes y mayores en una de las bases sobre la que se fundamenta la sociedad inclusiva, que promueve el intercambio, el diálogo, el apoyo mutuo y la solidaridad. En respuesta a esta cuestión ha habido en todos los países un interés cada vez más creciente por los programas intergeneracionales (PI), con el objetivo de acercar a las personas mayores y a los jóvenes, fuera del ambiente familiar, en torno a una serie de actividades planificadas.

Los PI han sido definidos de muy diversas maneras (Ventura-Merkel y Lidoff, 1983; McCrea et al., 2004, citados por Hatton-Yeo y Ohsako, 2001;

1. Este trabajo forma parte del Proyecto de Investigación «Ciudad y calidad de vida. El uso social de los espacios públicos abiertos en ciudades españolas» (CSO 2010-19007), financiado por el Plan Nacional de I+D+i del Ministerio de Ciencia e Innovación del Gobierno de España. 
Newman y Sánchez, 2007; VV. AA., 2008), pero todas las definiciones ${ }^{2}$ tienen en común que en un PI participan personas de distintas generaciones, implica actividades dirigidas a alcanzar fines beneficiosos para todas ellas y para la comunidad en la que viven, y que gracias al PI los participantes mantienen relaciones de intercambio. Su objetivo es que las prácticas intergeneracionales ayuden a construir comunidades más confiables y seguras, haciendo posible una disminución de las tensiones, de la mutua desconfianza y de la falta de respeto entre los mayores y los jóvenes (The Beth Johnson Foundation, 2010; Hatton et al., 2004; Zeldin et al., 2005; Pain, 2005, Sánchez et al., 2010).

Los PI comenzaron a implantarse en los Estados Unidos, en las décadas de los sesenta y los setenta, con la finalidad de acercar a las generaciones que se estaban distanciando. Posteriormente, hasta los años noventa, y también en Estados Unidos y Canadá, se utilizaron para abordar problemas sociales relacionados con necesidades culturales, sociales y económicas (Newman y Sánchez, 2007: 53). Después de esta década y hasta la actualidad, se han extendido las experiencias por todo el mundo desarrollado, y en algunos países en vías de desarrollo, sobre todo de América Latina (VV. AA., 2009; Romero et al., 2009; Lladó, 2010).

En los países de la UE se ha producido un incremento cada vez mayor de estos programas. Aparecen como respuesta a problemáticas determinadas, como la integración de las personas inmigrantes en Holanda, a cuestiones políticas relacionadas con la inclusión y los nuevos roles de las personas mayores en el Reino Unido; o con la percepción de una cierta crisis en los modelos de solidaridad familiar tradicionales y el interés por impulsar el envejecimiento activo, en el caso de España (Newman y Sánchez, 2007: 53).

En España, el IMSERSO puso en marcha en 2005 una red temática dedicada a la promoción de las relaciones intergeneracionales - Red Intergeneracional- (Giménez, 2013) y en 2010 publicó una guía para los programas intergeneracionales (Sánchez et al., 2010). Del mismo modo, todas las comunidades autónomas han desarrollado programas diversos, la mayor parte de ellos llevados a cabo en espacios cerrados - centros de día de mayores, colegios, centros ciudadanos, bibliotecas públicas, etc.

No obstante, los espacios públicos abiertos, en tanto que lugares de encuentro de usuarios diversos, poseen una gran potencialidad para facilitar la interrelación entre los distintos grupos de edad (Borja y Muxí, 2003; Schlack, 2007;

2. La definición de programa intergeneracional que hemos utilizado es la elaborada por los participantes en el curso "Actuaciones para promover las relaciones intergeneracionales», organizado por el IMSERSO y la Agencia Española de Cooperación Internacional al Desarrollo, celebrado en octubre de 2008 en La Antigua (Guatemala): «Los programas intergeneracionales son medios, estrategias, oportunidades y formas de creación de espacios para el encuentro, la sensibilización, la promoción del apoyo social y el intercambio recíproco, intencionado, comprometido y voluntario de recursos, aprendizajes, ideas y valores encaminados a producir entre las distintas generaciones lazos afectivos, cambios y beneficios individuales, familiares y comunitarios, entre otros, que permitan la construcción de sociedades más justas, integradas y solidarias» (VV. AA., 2008). 
García Doménech, 2013, citados por García y Martí, 2014), por lo que pueden llegar a convertirse en los escenarios idóneos en donde impulsar las relaciones intergeneracionales, siempre que su diseño lo estimule y los servicios sociales de las administraciones públicas y/o las asociaciones ciudadanas desarrollen estrategias y actividades para el encuentro y el intercambio recíproco de aprendizajes, ideas y valores (VV. AA., 2008). En definitiva, que la ciudad, a través del espacio público abierto, propicie y contribuya al logro de los objetivos de los programas intergeneracionales.

La ciudad debe, por tanto, prepararse para este nuevo reto, diseñando o renovando espacios que favorezcan la interrelación entre las generaciones, pues el modelo tradicional de la planificación urbana de las últimas décadas se ha centrado en la construcción de equipamientos específicos generacionales, lo que no favorece estas relaciones - centros de mayores, centros de juventud, residencias de mayores, etc.- (Enciso, 2012); y en el diseño de espacios públicos abiertos, en ocasiones, poco inclusivos por problemas de accesibilidad y/o de localización, y por la segmentación de las zonas destinadas a cada generación -zonas deportivas para jóvenes, bancos y aparatos de gimnasia para mayores, áreas de juego para niños...-, que impiden físicamente la interrelación. De ahí que sea indispensable que existan espacios en donde las generaciones interactúen, tanto en encuentros espontáneos, como en la realización de actividades planificadas que faciliten su interacción.

Conviene, por tanto, que las administraciones públicas locales se pongan al servicio de las relaciones entre generaciones, potenciando no solo programas intergeneracionales que se desarrollen en los espacios públicos abiertos, sino también acondicionando con equipamientos de uso común algunos de estos, para que se pueda producir la integración de las personas de diferentes edades de forma espontánea, como ya se ha hecho con las residencias intergeneracionales (García y Martí, 2014) y los centros intergeneracionales (Jarrot y Weintraub, 2007). Por ello, es necesaria la adecuación de espacios públicos abiertos en donde sea posible la interacción de las distintas generaciones en torno a recursos que posibiliten un ocio compartido -zonas para juegos de mesa, pista de petanca, campo de minigolf, pistas para el baile o gimnasia al aire libre, carpas para actividades relacionadas con la lectura, etc. - o, por lo menos, que no impidan la posibilidad de llevarlo a cabo. En los últimos años en muchas ciudades de países menos desarrollados - Santo Domingo y Quito en Ecuador, Soacha en Colombia, etc.- - y en algunas españolas - San Cristóbal de La Laguna en Tenerife (parque de La Vega), Santiago de Compostela en La Coruña (parque de Ramírez), Orense (Santa Eulalia de Beiro), Barakaldo en Vizcaya (parque de Okeluri), Berriozar en Navarra (parque Basoa), etc.se han construido parques que se catalogan de intergeneracionales al haberse habilitado zonas para niños, adolescentes y mayores. En realidad, responden más a un carácter claramente multigeneracional, pues si bien es posible la confluencia de todos los grupos etarios, los equipamientos están segregados por edades, lo que impide la interrelación, requisito indispensable para que se produzca la intergeneracionalidad. 


\section{Objetivos y metodología}

Con el objeto de aproximarnos a la potencialidad de los espacios públicos abiertos para el desarrollo de las relaciones intergeneracionales se indaga, en primer lugar, en las relaciones espontáneas entre jóvenes y mayores en dos espacios públicos del centro de la ciudad de Santa Cruz de Tenerife. Y en segundo lugar, se examinan los factores que contribuirían a impulsar la interacción entre ambos grupos de edad: los programas intergeneracionales y las características físicas que deben reunir los espacios públicos abiertos para facilitar la interacción entre las generaciones.

Para el análisis se ha empleado una metodología combinada, cuantitativa y cualitativa, similar a la aplicada en otras investigaciones referidas a los espacios públicos (Hopkins y Pain, 2007; Prats et al., 2012a y 2012b; Ortiz et al., 2014; Calero et al., 2014).

Las relaciones entre jóvenes y mayores en los espacios públicos abiertos se han analizado a partir del estudio del uso y la apropiación que ambos grupos de edad realizan en dos lugares del centro de la ciudad de Santa Cruz de Tenerife: la Alameda del Duque de Santa Elena y la plaza Weyler.

Para trabajar con los/las jóvenes se contactó con la dirección de dos colegios situados en el área central de la ciudad de Santa Cruz de Tenerife -el Colegio privado-concertado La Salle - San Ildefonso y el Instituto público de Enseñanza Secundaria Andrés Bello- con la finalidad de realizar una encuesta al alumnado ${ }^{3}$.

La información obtenida fue procesada con el software estadístico SPSS que facilitó la explotación descriptiva de los datos mediante tablas de contingencia en las que se entrecruzaron variables diversas, lo que permitió indagar sobre el interés de los jóvenes por los espacios públicos, cuáles eran los más frecuentados y el uso y apropiación que hacían de los mismos - los días y horas de afluencia, las variadas motivaciones que determinaban la concurrencia a los diferentes espacios públicos, la dimensión de los grupos de amistades, las actividades que desarrollaban, los lugares de estancia habituales y su percepción sobre el ambiente. Las relaciones con los adultos mayores se valoraron a partir de las preferencias para compartir los espacios, sus comportamientos, interacciones y posibles conflictos. En todos los aspectos analizados, el género se utilizó como variable transversal, pues puede introducir disparidades relevantes.

Estas informaciones cuantitativas se matizaron y profundizaron mediante técnicas cualitativas. En primer lugar se llevaron a cabo cuatro sesiones de entrevistas semiestructuradas a conjuntos de cinco o seis jóvenes, diferenciados por sexos — dos grupos de chicas y dos de chicos—, con el objetivo de que las respuestas no se vieran condicionadas por los roles de género. La finalidad era

3. La razón de trabajar con los adolescentes en los centros educativos en lugar de encuestarlos y entrevistarlos directamente en los espacios públicos, radica en la mayor predisposición de los jóvenes a colaborar en la investigación en el marco de una institución cuya dirección y profesorado colabora y la apoya como una actividad de aprendizaje. 
constatar la valoración de los y las jóvenes respecto de las personas de mayor edad y su disposición para participar en actividades de intercambio de experiencias y conocimientos, si se planificaran en los espacios públicos.

Una vez verificados que los dos espacios más frecuentados del centro de la ciudad eran La Alameda y la plaza Weyler, se sometieron a un proceso de observación sistemática. Para ello se realizaron un total de seis reconocimientos durante dos días laborables y un día festivo (mañana y tarde), con la finalidad de determinar la posible existencia de ritmos cambiantes en el uso. En las observaciones se incluyó la perspectiva de género con objeto de constatar la existencia de desigualdades en el uso y la apropiación del espacio entre varones y mujeres. Mediante fichas se registraron las condiciones físicas de las plazas, así como las actividades desarrolladas por los usuarios según el sexo y el grupo de edad, con la finalidad de determinar posibles variaciones, de género y generacionales, en los comportamientos. En todos los días se fotografiaron escenas sobre los usos y apropiación de los usuarios de los dos espacios públicos.

Posteriormente se llevaron a cabo sondeos de opinión a las personas mayores usuarias de La Alameda y la plaza Weyler con objeto de comprobar las motivaciones de su uso y su predisposición a compartir actividades comunes en los espacios públicos con personas jóvenes. Se partió de un guión preestablecido aunque se planteó de forma abierta a modo de conversación, lo que permitió enriquecerlo con las aportaciones de los participantes. Las cuestiones se estructuraban en torno a dos ejes conductores: las formas de uso - con preguntas sobre la frecuencia, el horario, el lugar de residencia, las razones del uso, etc.—, y su disponibilidad para compartir actividades con los/las jóvenes en los espacios públicos — con preguntas sobre la valoración sobre la juventud y su comportamiento en el espacio público. Se realizaron un total de veinte sondeos (once varones y nueve mujeres), en turnos de mañana y de tarde y en diferentes días. Las conversaciones fueron grabadas, previo consentimiento, y posteriormente transcritas.

A fin de completar el análisis de la potencialidad del espacio público para desarrollar relaciones intergeneracionales, se efectuaron varias entrevistas a diversos técnicos: a las coordinadoras del Programa de Mayores del Instituto Municipal de Atención Social (IMAS), con la finalidad de conocer los programas intergeneracionales que se están desarrollando, si algunos se celebraban en los espacios públicos, así como recabar sus opiniones sobre la posibilidad de llevarlas a la práctica en estos lugares; al responsable del proyecto municipal de participación ciudadana "Con Red» — cuyo objetivo es analizar el uso de los espacios públicos por parte de las asociaciones ciudadanas-, con objeto de recabar información sobre las experiencias de carácter intergeneracional llevadas a cabo por dichas asociaciones. Por último, se consultó con un experto urbanista, participante en el proyecto de remodelación de la Alameda de Duque de Santa Elena, sobre las características físicas y los tipos de equipamientos que deben reunir los espacios públicos abiertos para convertirse en intergeneracionales. 


\section{Los espacios públicos de observación: la Alameda del Duque de Santa Elena y la plaza Weyler}

Puesto que no todos los espacios públicos son iguales — situación en la ciudad, morfología, accesibilidad...- ni las generaciones se comportan siempre de igual manera - contexto social, cultural y económico-, las experiencias de jóvenes y mayores en los espacios públicos son de distinta naturaleza. De ahí que el análisis empírico reflejará la interacción intergeneracional en los dos espacios seleccionados de la ciudad de Santa Cruz de Tenerife: la Alameda del Duque de Santa Elena y la plaza Weyler. Ambos lugares son utilizados de forma intensiva por numerosos usuarios de características distintas, y en ellos, previamente, se ha constatado la presencia asidua de grupos de mayores y de jóvenes (Delgado y Calero, 2012; García Herrera et al., 2012).

Los dos espacios pertenecen al distrito Centro-Ifara de la ciudad de Santa Cruz de Tenerife. Desde el punto de vista urbano, es el más antiguo de la ciudad, un área consolidada a finales del siglo xix y que fue, de forma progresiva, densificándose a lo largo del xx. En ella coexisten un conjunto de sectores diferenciados desde el punto de vista estructural, morfológico y social que combina distintas apariencias y usos residenciales diversos. Esta amplia zona cuenta con un número relativamente reducido de espacios públicos abiertos, si tenemos en cuenta su elevada población y las altísimas densidades; un conjunto de plazas, plazoletas y paseos conforman la red de espacios públicos abiertos de mayor antigüedad y de más elevado valor simbólico de la ciudad, que incluso trasciende los límites capitalinos. Dentro de esta red, tanto la Alameda del Duque de Santa Elena como la plaza Weyler son dos de los espacios más interesantes y frecuentados por su accesibilidad y su posición medular en la estructura urbana de la ciudad. Se trata de lugares bien percibidos donde se mezclan dinámicas distintas derivadas de usos y usuarios diversos.

La Alameda del Duque de Santa Elena (Figura 1) se sitúa en la entrada noreste de la ciudad de Santa Cruz de Tenerife y fue construida en 1787. Su localización anexa a las dos plazas principales de la ciudad - la plaza de España y la de La Candelaria- y su proximidad al puerto contribuyeron, desde su inauguración, a que se convirtiera en un lugar muy frecuentado. En los primeros años del siglo XXI, experimenta una remodelación dentro de un proyecto de mayor envergadura que implicó la transformación de todo el frente litoral, cuyos objetivos fueron, de una parte, lograr un mayor contacto entre la ciudad y el puerto y, de otra, incrementar el atractivo turístico de Santa Cruz. El proyecto materializó no solo una profunda transformación morfológica, sino también posibilitó la peatonalización y continuidad entre esta y las dos plazas próximas - España y La Candelaria-, conformando un amplio conjunto unificado para el tránsito peatonal. En la remodelación, La Alameda recupera artificiosamente el arco suntuoso de entrada (Figura 1), que intenta individualizarla, mantiene su trazado rectangular, se le incorpora suelo de tierra batida, 


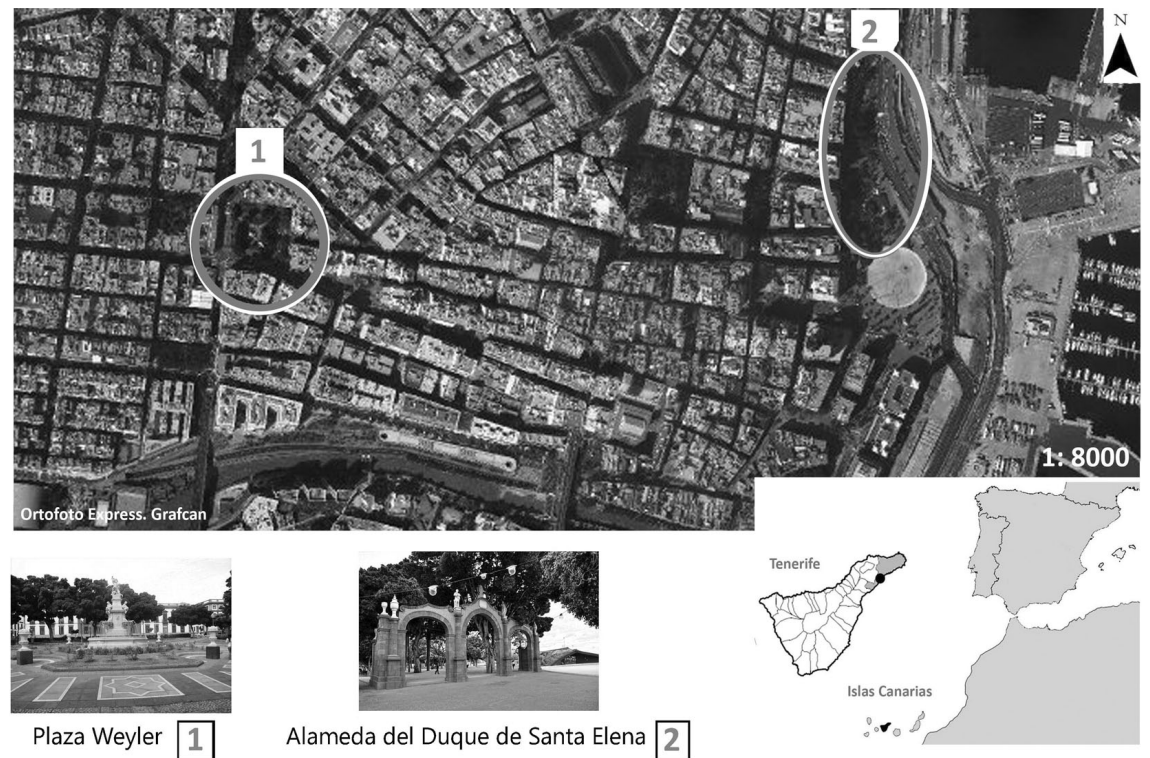

Figura 1. Situación de la Alameda del Duque de Santa Elena y la plaza Weyler.

Fuente: Grafcan. Elaboración propia.

mejora las condiciones de acceso y conserva el arbolado - insuficiente para las elevadas horas de sol que existen en la ciudad durante todo el año- - y bancos de madera dispersos. También se le han añadido dotaciones diversas como un parque infantil y un restaurante. Más que una rehabilitación se trata de una profunda renovación que le ha hecho perder singularidad y la ha convertido en una especie de apéndice del conjunto formado por las plazas de La Candelaria y la de España.

La plaza Weyler se localiza en el interior de la ciudad (Figura 1), se construyó en el último cuarto del siglo XIX, concretamente entre 1879 y 1897 y su diseño no ha experimentado modificación alguna posterior. Morfológicamente se trata de un recinto cuadrangular sin cerramiento, abierto y accesible, organizado por un conjunto simétrico de parterres ajardinados y arbolados, que ocupan un $40 \%$ de la superficie total, y separan calles o paseos interiores centralizados por la histórica fuente de mármol, el elemento arquitectónico de mayor relevancia. La plaza Weyler ha servido como lugar de esparcimiento del conjunto urbano que se articula en su entorno, pero su situación en la ciudad y su carácter de espacio de tránsito y de encuentro -un auténtico intercambiador urbano para los habitantes y visitantes de Santa Cruz - han ocasionado que la afluencia de usuarios no solo sea elevada sino que, también, la procedencia de los mismos sobrepase los límites de su área de influencia urbana. 


\section{Las relaciones intergeneracionales en el espacio público}

El uso y la apropiación que jóvenes y mayores hacen de los espacios públicos analizados difieren notablemente.

Los jóvenes, tanto chicos como chicas, presentan comportamientos similares: frecuentan La Alameda - sobre todo los que viven en el distrito Centro- en horario de tarde-noche y durante los fines de semana y días festivos; se reúnen en pandillas de cuatro o de seis componentes y sus lugares preferidos son la zona de los bancos (Figura 2) y el bar; las amistades proceden de su colegio $(59,2 \%)$ o de otros lugares $(37,1 \%)$, (Tabla 1$)$; y la estancia suele ser prolongada. En las formas de apropiación es donde se observa diferencias por género, pues, mientras la actividad más habitual de las chicas es la charla (79\%), los varones combinan la charla $(50 \%)$ con la práctica del skate $(50 \%)$. Su condición de espacio central explica la diversidad de usuarios jóvenes, pues funciona también como punto de reunión de jóvenes que viven en diferentes sitios de la ciudad.

No obstante, la profunda renovación que ha experimentado el lugar y las nuevas formas en que los jóvenes lo utilizan y se apropian de él, ha ocasionado la pérdida de identidad de este espacio singular para esta generación, como lo atestigua que más de las tres cuartas partes desconozcan su nombre real, reconociéndolo con el nombre de «El Plano» —en referencia a la topografía llana y a la jerga de los skaters-, y en menor proporción por «el Charco», «la Charca» o «la Fuente»—en clara referencia al lago artificial de la plaza de España, colindante con La Alameda-, e incluso confundan su denominación con la plaza de España.

Las personas mayores, en cambio, frecuentan muy poco La Alameda, y los que lo hacen viven en zonas relativamente próximas y, por tanto, se desplazan caminando. Van de mañana y permanecen entre media hora y hora y media; aunque por las tardes hay algunas mujeres mayores que cuidan de sus nietos en la zona de juego infantil. La utilizan como un lugar de paso, como zona de descanso (Figura 2) en un paseo más largo o para reunirse con alguna amistad. Valoran mucho la seguridad del lugar, la accesibilidad, la existencia de arbolado
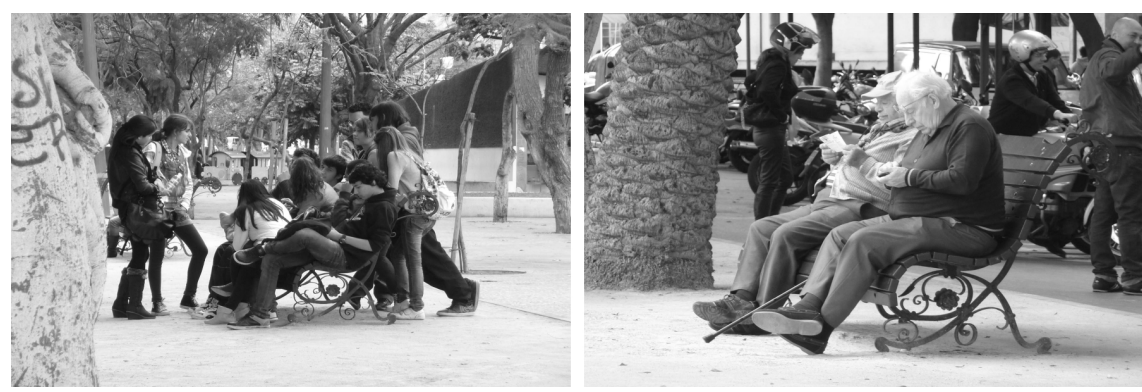

Figura 2. Jóvenes y mayores en La Alameda, relación entre iguales.

Fuente: Alejandro Armas Díaz. 
Tabla 1. Porcentajes más significativos sobre algunos aspectos del uso y la apropiación que hacen los y las jóvenes de la Alameda del Duque de Santa Elena

\begin{tabular}{|c|c|c|c|c|c|c|c|c|c|c|c|}
\hline \multicolumn{3}{|c|}{ Motivos para acudir } & \multirow{2}{*}{$\begin{array}{c}\begin{array}{c}\text { Días } \\
\text { de visita }\end{array} \\
\begin{array}{l}\text { Fines de } \\
\text { semana }\end{array}\end{array}$} & \multicolumn{3}{|c|}{$\begin{array}{c}\text { Procedencia } \\
\text { de las amistades }\end{array}$} & \multicolumn{3}{|c|}{$\begin{array}{l}\mathrm{N}^{\circ} \text { de amigos/as que } \\
\text { componen los grupos }\end{array}$} & \multicolumn{2}{|c|}{$\begin{array}{l}\text { Actividades } \\
\text { más frecuentes }\end{array}$} \\
\hline Cercanía & $\begin{array}{l}\text { Lugar de } \\
\text { reunión }\end{array}$ & Ambiente & & Colegio & $\begin{array}{c}\text { Otros } \\
\text { lugares }\end{array}$ & Barrio & 2 a 3 & 4 a 6 & Variable & Charla & Skating \\
\hline 39,3 & 39,3 & 21,4 & 92,1 & 59,2 & 37,1 & 3,7 & 32,5 & 43,2 & 24,3 & 57,9 & 42,1 \\
\hline
\end{tabular}

* Los porcentajes se han calculado sobre el total de respuestas válidas.

Fuente: encuestas al alumnado de $2^{\circ}$ curso de la ESO y $2^{\circ}$ curso de Bachillerato del Colegio La Salle San Ildefonso y El Instituto Andrés Bello de Santa Cruz de Tenerife realizadas en 2014. Elaboración propia.

Tabla 2. Porcentajes más significativos sobre algunos aspectos del uso y la apropiación que hacen los y las jóvenes de la plaza Weyler

\begin{tabular}{|c|c|c|c|c|c|c|c|c|c|c|}
\hline \multicolumn{3}{|c|}{ Motivos para acudir } & \multirow{2}{*}{$\begin{array}{c}\begin{array}{c}\text { Días } \\
\text { de visita }\end{array} \\
\begin{array}{l}\text { Fines de } \\
\text { semana }\end{array}\end{array}$} & \multicolumn{3}{|c|}{$\begin{array}{c}\text { Procedencia } \\
\text { de las amistades }\end{array}$} & \multicolumn{3}{|c|}{$\begin{array}{l}\mathrm{N}^{0} \text { de amigos/as que } \\
\text { componen los grupos }\end{array}$} & \multirow{2}{*}{$\begin{array}{c}\begin{array}{c}\text { Actividad más } \\
\text { frecuente }\end{array} \\
\begin{array}{c}\text { Punto de } \\
\text { encuentro }\end{array}\end{array}$} \\
\hline Cercanía & $\begin{array}{l}\text { Lugar de } \\
\text { encuentrc }\end{array}$ & Ambiente & & Colegio & $\begin{array}{c}\text { Otros } \\
\text { lugares }\end{array}$ & Barrio & 2 a 3 & 4 a 6 & Variable & \\
\hline 25,0 & 50,0 & 25,0 & 87,5 & 42,9 & 42,8 & 14,3 & 40,0 & 20,0 & 40,0 & 75,0 \\
\hline
\end{tabular}

* Los porcentajes se han calculado sobre el total de respuestas válidas.

Fuente: encuestas al alumnado de $2^{\circ}$ curso de la ESO y $2^{\circ}$ curso de Bachillerato del Colegio La Salle San Ildefonso y El Instituto Andrés Bello de Santa Cruz de Tenerife realizadas en 2014. Elaboración propia.

que les permite descansar a la sombra, y que, en general, la zona está bien equipada; aunque algunos/as la consideran ruidosa - tráfico circundante, juegos infantiles, presencia de adolescentes que practican skating - y se quejan de la inexistencia de aseos. Sin embargo, los que la conocían con anterioridad, muestran su disconformidad con la transformación que ha experimentado — sobre todo el suelo de tierra—, añoran el diseño tradicional, por considerarlo más acogedor y recuerdan con nostalgia «Los Paragüitas» — denominación popular de La Alameda en referencia a la terraza del antiguo bar- que proporcionaba al lugar un ambiente muy animado.

Así, mientras el grupo de mayores manifiesta cierta añoranza por La Alameda anterior a la renovación, los jóvenes se apropian del modernizado espacio, cambian su nombre y contribuyen a la construcción de una nueva identidad.

La plaza Weyler, por su parte, es un espacio menos frecuentado por jóvenes (21\%), al que acuden más los chicos $(66,7 \%)$ que las chicas $(33,7 \%)$, y más de la mitad viven en el centro de la ciudad, algunos muy cerca del lugar. Se mantienen las mismas pautas que observamos para La Alameda, en relación con el predominio del uso de este espacio público los fines de semana y en jornadas de tarde y tarde-noche y la procedencia de las amistades (Tabla 2).

La plaza funciona para los chicos y las chicas, sobre todo, como punto de encuentro (Tabla 2 y Figura 3) para trasladarse a otros lugares de la ciudad; 

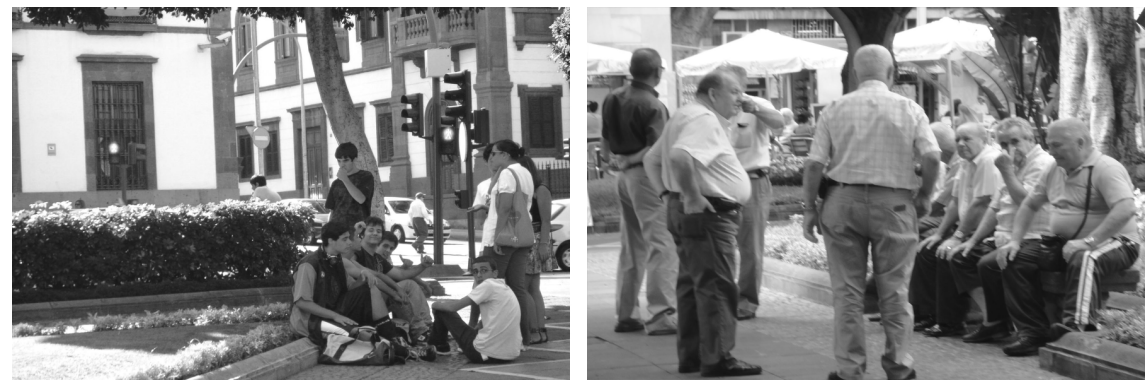

Figura 3. Jóvenes y mayores en la plaza Weyler, la indiferencia intergeneracional.

Fuente: Carmen Gloria Calero y Carmen Rosa Delgado.

uno de ellos es La Alameda, bien conectada con la plaza Weyler por la céntrica calle de El Castillo - la arteria más antigua e importante de Santa Cruz. Se concentran en los bancos, en la esquina de la plaza más próxima a la parada del tranvía y en la escalinata que le da salida por el sur. La plaza se considera un lugar seguro y se percibe como un buen espacio de relación, aunque manifiestan que debería tener un mejor mantenimiento y equipamientos en general.

Por su parte, las personas mayores, fundamentalmente varones, acuden habitualmente a la plaza Weyler por las mañanas, su estancia es prolongada, pasando en ella de dos a tres horas y la eligen por ser céntrica y concurrida. Para este colectivo la plaza es un lugar de encuentro y de relación entre iguales. Los usuarios son generalmente vecinos del centro de Santa Cruz que se desplazan caminando, aunque los que proceden de barrios periféricos utilizan los medios de transporte colectivo. Algunos, incluso, llegan a ella después de un largo paseo en el que han frecuentado algún otro espacio público. Ocupan los bancos y suelen preferir aquellos situados en la zona este, la menos ruidosa y más alejada de la parada del tranvía. Las actividades más usuales son la charla con su habitual grupo de amistades (Figura 3) — seis o más personas- y la observación del paso de la gente. Les gusta el ambiente en general pero critican el mobiliario urbano, los bancos de piedra-anticuados y escasamente ergonómicos-, la ausencia de aseos públicos y las escasas zonas de sombra (Delgado y Calero, 2012).

La Alameda y la plaza Weyler son, por tanto, espacios públicos que permiten la coexistencia de jóvenes y mayores, pero los usos que ambas generaciones hacen de ellos son diferentes. Varían en los horarios de visita: los mayores - sobre todo los varones - la frecuentan durante todos los días $y$, fundamentalmente, en horario de mañana, mientras los jóvenes - tanto chicos como chicas - lo hacen de tarde-noche y durante los fines de semana y festivos. Difieren también en la manera en la que acuden a los espacios públicos, pues los mayores suelen ir solos - a reunirse o no con sus amistades - o acompañados por sus parejas o cuidadores; en cambio, la relación de los jóvenes con el espacio público no se puede desligar de su círculo de 
Tabla 3. Porcentajes más significativos de las valoraciones que hacen los y las jóvenes sobre su relación con las personas mayores en la Alameda del Duque de Santa Elena

\begin{tabular}{|c|c|c|c|c|c|c|c|}
\hline \multicolumn{2}{|c|}{$\begin{array}{l}\text { Preferencia por compartir } \\
\text { con su misma generación }\end{array}$} & \multicolumn{2}{|c|}{$\begin{array}{c}\text { No tienen relación con las } \\
\text { personas mayores }\end{array}$} & \multicolumn{2}{|c|}{$\begin{array}{l}\text { Inexistencia de relación } \\
\text { por sus conductas } \\
\text { inadecuadas }\end{array}$} & \multicolumn{2}{|c|}{$\begin{array}{l}\text { No tienen conflictos con } \\
\text { mayores }\end{array}$} \\
\hline Varones & Mujeres & Varones & Mujeres & Varones & Mujeres & Varones & Mujeres \\
\hline 50,0 & 19,2 & 94,3 & 95,0 & 66,7 & 62,5 & 41,0 & 59,0 \\
\hline
\end{tabular}

Tabla 4. Porcentajes más significativos de las valoraciones que hacen los y las jóvenes sobre su relación con las personas mayores en la plaza Weyler

\begin{tabular}{|c|c|c|c|c|c|c|c|}
\hline \multicolumn{2}{|c|}{$\begin{array}{l}\text { Preferencia por compartir } \\
\text { con su misma generación }\end{array}$} & \multicolumn{2}{|c|}{$\begin{array}{c}\text { No tienen relación con las } \\
\text { personas mayores }\end{array}$} & \multicolumn{2}{|c|}{$\begin{array}{l}\text { Inexistencia de relación } \\
\text { por sus conductas } \\
\text { inadecuadas }\end{array}$} & \multicolumn{2}{|c|}{$\begin{array}{l}\text { No tienen conflictos con } \\
\text { mayores }\end{array}$} \\
\hline Varones & Mujeres & Varones & Mujeres & Varones & Mujeres & Varones & Mujeres \\
\hline 30,7 & 12,5 & 84,6 & 83,3 & 80 & 66,7 & 35,3 & 64,7 \\
\hline
\end{tabular}

* Los porcentajes se han calculado sobre el total de respuestas válidas.

Fuente: encuestas al alumnado de $2^{\circ}$ curso de la ESO y $2^{\circ}$ curso de Bachillerato del Colegio La Salle San Ildefonso y El Instituto Andrés Bello de Santa Cruz de Tenerife realizadas en 2014. Elaboración propia.

amistades, el grupo es el que le da sentido y contribuye a forjar su identidad (Prats et al., 2012b).

Puesto que los diseños de ambos espacios públicos son diferentes, las formas de apropiación de ambas generaciones también difieren. La Alameda es un lugar de estancia prolongada, donde la charla y el deporte en los chicos — skate — son las más relevantes. Para los mayores, en cambio, es un lugar de tránsito y de descanso puntual, el encuentro solo se da en algún caso de forma excepcional. Por el contrario, la plaza Weyler funciona en sentido inverso. Para los mayores es un lugar de interacción entre iguales y de estancia prolongada de grupos que encuentran este lugar, bullicioso, concurrido y muy atractivo. En contraposición, para los jóvenes la plaza funciona como lugar de encuentro para desplazarse hacia otros espacios, permaneciendo en ella poco tiempo; su posición céntrica permite conectar con el resto de los espacios públicos del distrito Centro.

En lo que respecta a las relaciones intergeneracionales, tanto en La Alameda como en la plaza Weyler los jóvenes manifiestan mayoritariamente no tener relación alguna con los mayores (Tablas 3 y 4).

En La Alameda, las diferencias entre chicos y chicas solo se manifiestan en que los primeros prefieren los lugares en donde solo acudan personas de su misma generación; las chicas, en cambio, parecen no dar tanta importancia a la presencia en el espacio de otros grupos etarios (Tabla 3). El resto de los comportamientos no difieren, pues cuando se trata de buscar las causas de la escasa 
interacción positiva, tanto varones como mujeres la atribuyen a sus conductas inadecuadas y a la intransigencia de los mayores. En muchos casos, la escasa relación se traduce en forma de conflictos puntuales, sobre todo entre los varones de ambas generaciones, ya sea por llamadas de atención ante actuaciones que subvierten las normas sociales o por los inconvenientes que les supone a los mayores la práctica del skating.

Esta falta de relaciones entre jóvenes y mayores y las diferencias por sexos en las valoraciones se repiten de forma simétrica en la plaza Weyler (Tabla 4), lo que puede revelar que son los comportamientos generacionales arraigados, el desapego y cierta intolerancia lo que impide unas relaciones intergeneracionales fluidas y espontáneas y que el diseño del espacio o su adecuación no es una variable determinante.

Los dos grupos de edad - tanto varones como mujeres - manifiestan, por tanto, no relacionarse en ninguno de los espacios analizados, a pesar de que, en ocasiones, coinciden en determinadas franjas horarias o en los periodos vacacionales. Para las personas jóvenes, los espacios públicos son los lugares de la amistad entre iguales y no sienten interés alguno por interactuar, al igual que les sucede a los mayores (Figuras 2 y 3 ), pues sus inquietudes son diferentes y los estereotipos sociales y los comportamientos más extendidos disuaden la relación. Los jóvenes se sienten más cómodos entre personas de su misma edad, consideran que los mayores los juzgan de forma inapropiada, se sienten observados y criticados por su modo de vestir o peinar, e incluso algunos consideran que los espacios públicos deberían estar segregados por edades. «Con los señores mayores algunas cosas no las puedes hacer, que haces con tus colegas. Cada espacio público tiene su público» $\left(\mathrm{Sergio}^{4}\right)$.

Por su parte, los mayores, en general, mantienen con los jóvenes una actitud distante pues consideran que no son valorados ni tenidos en cuenta y que son irrespetuosos. «(...) los jóvenes pasan de nosotros. Van por su lado» (Francisco).

La escasa interacción entre ambos grupos de edad se produce en forma de conflictos puntuales cuando los y las mayores reprochan a los y las jóvenes conductas que consideran inadecuadas o incívicas. "[...] Se ponen a comer pipas y dejan ahí el suelo todo sucio» (Ismael); «algunas veces les llamamos la atención por el tratamiento de los jardines y los jóvenes responden ¡qué le pasa al pureta [viejo] ese!» (Vicente).

\section{Programas intergeneracionales y espacio público abierto}

A pesar de que cada vez más se ha generalizado la práctica intergeneracional en todos los países a partir de los programas intergeneracionales, ha habido poca investigación sobre las relaciones entre jóvenes y mayores en el espacio público abierto (Pain, 2005; Vanderbeck, 2007; Lladó, 2010). Sin embargo, dichos espacios — plazas, parques, paseos— se presentan como los más favorecedores,

4. Se utilizan pseudónimos para mantener el anonimato de las personas entrevistadas. 
no solo de la coexistencia entre las distintas edades, sino también de la interrelación y el intercambio de saberes y experiencias, de aprendizajes diversos y de consolidación de conductas ciudadanas positivas. «La propia razón de ser del espacio público acredita su uso como vehículo de expresión ciudadana, cohesión social e integración intergeneracional» (Borja y Muxí, 2003; Schlack, 2007; García Doménech, 2013, citado por García y Martí, 2014: 66).

En los últimos años, se han ido incrementando en España las actividades intergeneracionales desarrolladas en los espacios públicos abiertos y son ya muchos los municipios - Madrid, Oviedo, Zaragoza, Granada, Sevilla, Vigo, Estepona, etc.- que llevan a cabo este tipo de experiencias, sobre todo en los parques. Se trata de variadas actividades de carácter esporádico, potenciadas desde los servicios sociales municipales para potenciar el envejecimiento activo. En su mayor parte son de aprendizaje, diversión, ocio o deporte en las que los mayores, jóvenes y niños se prestan mutuo servicio.

En Santa Cruz de Tenerife, el Instituto Municipal de Atención Social (IMAS), viene desarrollando desde el año 2000 varios proyectos de intergeneracionalidad organizados por el Negociado de Mayores (Geas7, 2011), pero son muy pocas las experiencias llevadas a cabo en espacios públicos abiertos y ninguna de ellas en los que han sido objeto del presente análisis. Coincidiendo con la conmemoración de días señalados, se ha celebrado «El día de la familia» —en que abuelos/as, hijos/as y nietos/as se reúnen en la céntrica plaza del Príncipe con la finalidad de fomentar la interrelación y comunicación entre las distintas generaciones de una misma familia; $\mathrm{y}$ «El día del vecino» celebrado en el barrio de Ofra con el objetivo de establecer lazos y relaciones entre ciudadanos. Excepción hecha de estas celebraciones puntuales, los proyectos están claramente dirigidos a las personas mayores, por lo que no pueden ser considerados de intergeneracionalidad; el interés principal es conseguir que el colectivo de mayores se integre en actividades relacionadas con programas de salud y de envejecimiento activo.

El diseño de una práctica intergeneracional, no solo debe tener en cuenta los objetivos sociales previstos, sino también el espacio físico en que se va a desarrollar y si este se presta o no a dichas relaciones. Junto a los programas intergeneracionales, es imprescindible que dicho espacio permita las relaciones entre los distintos grupos de edad. La falta de lugares comunes para jóvenes y mayores - entendidos como espacios de encuentro y conocimiento recíproco-, favorece los procesos de aislamiento respecto de los otros, la dificultad de diálogo y la indiferencia. Pues bien, de los dos espacios analizados, la plaza Weyler presenta un diseño abigarrado con falta de espacios libres y la Alameda del Duque de Santa Elena, en cambio, cuenta con una amplia zona de uso común.

A la vista de que en Santa Cruz de Tenerife no se dan relaciones intergeneracionales en los espacios públicos abiertos de forma espontánea y tampoco se han proyectado programas específicos, el análisis de su potencialidad como espacios de intergeneracionalidad se hará con un enfoque prospectivo, a partir de las opiniones de las personas jóvenes, de las mayores y de los expertos. 
De las entrevistas realizadas a los y las adolescentes se deduce una relativa disponibilidad para relacionarse con las personas mayores en los espacios públicos en actividades bidireccionales, de ocio y de aprendizaje, en las que ambos grupos generacionales muestren sus habilidades y conocimientos. «Con tu grupo de amigos y con un grupo de mayores, seguro que interactúas y te reirías [...]» (Ale). Por su parte, los sondeos de opinión realizados a las personas mayores - sobre todo a los varones, por ser el colectivo que más frecuenta los espacios públicos analizados- muestran un mayor entusiasmo por el entretenimiento que podrían reportarles las actividades intergeneracionales en los espacios públicos — por lo que supone de ruptura de la rutina diaria—, más que por las posibilidades de intercambio de conocimientos entre ambos grupos, pues parten del convencimiento de que a los/las jóvenes no les interesan sus opiniones. "Yo creo que los mayores participarían más que los jóvenes, ellos viven en su mundo, en su tecnología y la relación humana se ha perdido» (Moisés).

Ambos grupos de edad muestran, en principio, predisposición para actuar en el espacio público en actividades intergeneracionales. Entre el colectivo de jóvenes hay diferencias por género, y si bien las chicas participarían en cualquier iniciativa, los chicos lo harían solo en las que fueran de su interés y, sobre todo, si ellos son los transmisores de habilidades. El grupo de mayores expresa mayor disposición a aceptar cualquier tipo de experiencia intergeneracional siempre que le suponga entretenimiento, aunque manifiestan gran desconfianza hacia los jóvenes.

Las técnicas responsables del Negociado de Mayores, dejaron entrever los inconvenientes que supone el uso del espacio público abierto para actividades intergeneracionales que reúnan a mayores y jóvenes, debido a los equipamientos y características morfológicas de dichos espacios: el amueblamiento, especialmente las zonas de descanso y los aseos son imprescindibles, así como la posibilidad de usar alguna zona de forma intermitente donde colocar carpas que permitan realizar actividades. Reconocieron que los parques serían por su amplitud y posibilidades de aprendizaje — estatuas de personajes célebres, monumentos artísticos, vegetación, jardines - los lugares más idóneos para llevarlas a cabo. De los dos espacios analizados consideraron que La Alameda podría ser un lugar interesante para acoger algunas iniciativas de tipo cultural - por ejemplo, alguna exposición de fotografías antiguas en la que mayores voluntarios enseñen a jóvenes de los centros escolares la evolución histórica de este espacio emblemático de la ciudad y de su entorno-; y algunos torneos lúdicos - ajedrez, por ejemplo—, también en colaboración con los colegios. En cambio, la plaza Weyler no sería un lugar idóneo debido a sus reducidas dimensiones y a un diseño inapropiado.

Por su parte, el responsable del proyecto municipal de participación ciudadana «Con Red» —que trabaja desde el año 2014 el uso de los espacios públicos abiertos por parte de diferentes asociaciones ciudadanas- manifiesta que en ellos se desarrollan numerosas y variadas actividades, que aunque no hayan sido programadas con carácter intergeneracional, consiguen que se den 
relaciones entre jóvenes y mayores de forma espontánea, más horizontales, menos dirigidas y con resultados positivos. Considera que cuando desde las asociaciones ciudadanas se programan acciones encaminadas a solucionar problemas concretos, como organizar algún evento - fiestas patronales- o desarrollar algún pequeño proyecto comunitario - huertos urbanos-, los jóvenes y los mayores interactúan espontáneamente sin que sea necesario programar actividades intergeneracionales; las relaciones intergeneracionales, «deben surgir espontáneamente [...]. Los proyectos comunitarios deben perseguir solucionar problemas, y en el camino, durante el proceso, es cuando se establecen estas relaciones" " (Juan). Asimismo, considera que el lugar ideal para el desarrollo de las relaciones entre las generaciones es el espacio público abierto, «es el sitio» (Juan), pues en los cerrados, en ocasiones, los conflictos por la apropiación por parte de las generaciones impiden la interacción. En cambio, «el espacio público abierto, al ser el lugar de todos, los distintos individuos pueden relacionarse siempre que exista un proyecto comunitario común» (Juan).

Los espacios públicos que reúnan condiciones apropiadas pueden, por tanto, contribuir a favorecer la inclusión social, la cohesión, la ciudadanía y el desarrollo comunitario, si en ellos se proyectaran actividades de interés para ambos grupos generacionales.

En este sentido, el urbanista entrevistado considera que «el espacio físico es lo menos importante, no debe molestar, no puede impedir cualquier actividad» (Sergio) y no comparte la tendencia de crear espacios ex profeso de carácter intergeneracional. Es contrario a la obsesión por el diseño y la planificación: «no hay nada peor que una planificación que no tenga en cuenta las características del lugar y del ciudadano» (Sergio). Piensa que lo realmente importante es que el espacio público integre elementos de confort: sea atractivo, cómodo y seguro; e insiste en que la movilidad dinamiza al espacio, por lo que el recorrido es el que debe vertebrar el espacio público abierto y articular el conjunto de los equipamientos que permitan actividades diferentes para todas las generaciones, «el espacio debería planificarse como un recorrido desde donde se pueda acceder a elementos distintos» (Sergio). Considera que equipamientos destinados al juego — petancas, mesas de juego, etc. — son proclives a ser usados por todas las generaciones de forma espontánea, aunque lo realmente importante para conseguir la intergeneracionalidad no son los equipamientos, sino la presencia de animadores sociales que la incentiven; es decir, trasladar al sector público las iniciativas implementadas en las instalaciones hoteleras.

Respecto a los dos espacios de estudio, manifiesta su disconformidad con el diseño decimonónico de la plaza Weyler, al considerarlo inapropiado y limitante para las relaciones intergeneracionales, por lo que requeriría una renovación urgente «si observas la circulación de la población, el movimiento que hace no tiene nada que ver con el diseño y, siendo un lugar potente, gracias a

5. Este es el mismo planteamiento de Giménez (2013: 47-48) «el enfoque comunitario, clave para otros aspectos de la vida social en democracia, lo es para también para que sea posible el encuentro y la cooperación intergeneracional». 
su localización, no permite la realización de ninguna actividad [...] es necesario mejorar este espacio para las nuevas necesidades sociales ya que tiene un potencial importante para realizar actividades intergeneracionales» (Sergio). En cambio, La Alameda permite incluir elementos como mercadillos móviles o carpas que potenciarían las relaciones entre las generaciones.

En suma, de los dos espacios públicos analizados de la ciudad de Santa Cruz de Tenerife, por su extensión y estructura solo la Alameda del Duque de Santa Elena sería un lugar apropiado en donde se podrían experimentar prácticas de intergeneracionalidad de tipo cultural y de ocio, o donde se podrían poner en práctica proyectos no estrictamente intergeneracionales que, sin embargo, conducirían a este tipo de relaciones por su carácter abierto y motivador.

\section{Conclusiones}

La construcción de una sociedad inclusiva que propicie el intercambio, el diálogo y el apoyo mutuo entre los diversos grupos de edad debería ser entendida como una necesidad por el proceso de envejecimiento que afecta a las poblaciones actuales. Favorecer el desarrollo de las relaciones intergeneracionales supone un desafío social, que adquiere diferentes dimensiones y que se convierte en un reto urbano cuando estas relaciones encuentran en los espacios públicos lugares donde potencialmente pueden desarrollarse.

En los espacios centrales analizados de Santa Cruz de Tenerife, jóvenes y mayores no llevan a cabo relaciones intergeneracionales de forma espontánea, pues lo que predomina es una indiferencia mutua que el propio ciclo vital de cada grupo agudiza. Para los jóvenes, los espacios públicos son los lugares de la amistad entre iguales y, a priori, no sienten interés alguno por interactuar. Lo mismo les sucede a los mayores, pues sus inquietudes son diferentes y sus comportamientos más extendidos impiden la relación e incluso pueden favorecer la intolerancia y el desinterés por el otro, llegándose a generar el conflicto. Aunque los espacios públicos son lugares de encuentro, lo cierto es que la desconfianza mutua y los estereotipos sociales han generado una brecha generacional que dificulta, en estos lugares, las interrelaciones entre jóvenes y mayores.

Por otra parte, puesto que no se han programado para estos espacios estrategias intergeneracionales ni proyectos comunitarios, no se ha podido valorar su idoneidad como lugares de encuentro entre las generaciones. Aun así, tanto jóvenes y mayores, como los diversos técnicos entrevistados, reconocen la potencialidad de los mismos; la Alameda del Duque de Santa Elena en su actual diseño y la plaza Weyler si es remodelada de acuerdo a las necesidades de la sociedad actual.

Si los espacios públicos abiertos son los lugares de reunión habitual de usuarios de diferentes edades, de sociabilidad, de esparcimiento y de la relación por excelencia, podrán ser también los del intercambio de experiencias y conocimientos entre grupos generacionales distintos. Para que estas nuevas relaciones vayan apareciendo y se consoliden, es necesario que las administra- 
ciones públicas locales se impliquen en dos aspectos esenciales; por una parte, los espacios públicos abiertos deben ser entendidos como lugares de inclusión, de intercambio, favorecedores de actividades que involucren a las distintas generaciones en procesos de interrelación. Para ello es imprescindible que su diseño vaya dirigido a la generalidad, a todas las edades, y que sea capaz de facilitar la interacción entre grupos distintos mediante equipamientos o zonas de ocio de uso común. Por otra parte, es indispensable que los servicios sociales de las administraciones locales y las asociaciones ciudadanas pongan en marcha estrategias que favorezcan la relación intergeneracional, mediante programas concretos o actividades colaborativas en el espacio público que animen, de forma inducida o espontánea, a los diversos grupos de edad. De ese modo, la ciudad contribuiría, en su medida, a cumplir el objetivo de una «sociedad para todas las edades».

En definitiva, para que la relación intergeneracional sea posible en los espacios públicos abiertos, se requiere, especialmente, el desarrollo de actividades de encuentro entre los grupos de edad, ya sean promovidas a partir de programas intergeneracionales con la presencia de animadores sociales, inducidas por proyectos comunitarios que impliquen a todas la generaciones, o surjan de forma espontánea, favorecidas por un diseño apropiado del espacio con presencia de equipamientos y zonas comunes que fomenten la interacción.

\section{Referencias bibliográficas}

Borja, Jordi y Muxí, Zaida (2003). El espacio público. Ciudad y ciudadanía. Barcelona: Electa.

Calero Martín, Carmen Gloria; Delgado Acosta, Carmen Rosa y Armas Díaz, Alejandro (2014). «Espacio público, conflicto y convivencia: la plaza Primero de Mayo en Santa Cruz de Tenerife (Canarias)». Scripta Nova. Revista electrónica de Geografia y Ciencias Sociales, 18, 463-499. <http://www.ub.edu/geocrit/sn/sn-476. htm> [consulta: 26 de enero de 2015].

Delgado Acosta, Carmen Rosa y Calero Martín, Carmen Gloria (2012). «Personas mayores y uso de los espacios públicos. Un estudio de caso en la ciudad de Santa Cruz de Tenerife». En: Actas del XIX Coloquio de Historia Canario-Americana, 721-733.

Enciso Cobarros, Begoña (2012). Informe. Por el fomento de las relaciones intergeneracionales. IMSERSO, UDP. <http://www.mayoresudp.org/bddocumentos/ Informe- $4 \% \mathrm{C} 2 \% \mathrm{BA}$.-Por-el-fomento-de-las-Relaciones-Intergeneracionales.pdf> [consulta: 16 de junio de 2014].

García, Sergio y Martí, Pablo (2014). «Intergenerational Architecture and Public Space». ARQ (Santiago), 86. $<$ http://dx.doi.org/10.4067/S0717-69962014000100009>

García dOMÉnech, Sergio (2013). Reflexiones urbanas sobre el espacio público de Alicante. Una interpretación de la ciudad y sus escenarios. Alicante: Publicaciones Universidad de Alicante.

García-Herrera, Luz-Marina; García García, Antonio; Díaz Rodríguez, María del Carmen y Armas Díaz, Alejandro (2012). «Experiencias de ordenación y dinámicas sociales en espacios públicos centrales: las alamedas de Sevilla y de 
Santa Cruz de Tenerife». En: Miramontes, Angel; Royé, Dominic y Vila, Jose Ignacio (coords.). Las ciudades y el sistema urbano. Reflexiones en tiempos de crisis. Santiago de Compostela: Meubook, 131-141. <http:/www.uib.es/ggu/actes/ actas_urb_2012.pdf> [consulta: 16 de junio de 2014].

Geas 7. Consultoria Social (2011). I Plan Estratégico Municipal para las personas mayores de Santa Cruz de Tenerife. Servicio de Atención Social, Instituto Municipal de Atención Social (IMAS), Ayuntamiento de Santa Cruz de Tenerife. <http:// www.santacruzdetenerife.es/servicios-municipales/atencion-social/servicios/mayores/> [consulta: 2 de julio de 2014].

Giménez, Carlos (2013). «Lo intergeneracional, los esfuerzos por una sociedad más justa y las organizaciones no gubernamentales». En: PeÑa-López, Ismael; Zubero, Imanol, Giménez, Carlos y Arnanz, Enrique (2013). Ciudadanía y ONG. El nuevo papel del Tercer Sector ante el cambio de época. Barcelona: Fundación Esplai, 41-52. <http://www.ciudadaniayong.org/images/PDF/llibre_castella_ ciudadania_y_ONG.pdf> [consulta: 26 de enero de 2015].

Hatton-Yeo, Alan y Oнsako, Toshio (eds.) (2001). Programas intergeneracionales: politica pública e implicaciones de la investigación una perspectiva internacional. Hamburg: The Beth Johnson Foundation, Instituto de la Unesco para la Educación. <http://www.unesco.org/education/uie/pdf/intergenspa.pdf> [consulta: 16 de mayo de 2014].

Hatton-Yeo, Alan y Watkins, Celeste (2004). «Intergenerational Community Development. A practice guide». The Beth Johnson Foundation. <http://extension.psu.edu/ youth/intergenerational/program-areas/community-planning-visioning/intergenerational-community-development-...-a-practice-guides [consulta: 5 de junio de 2014].

Hopkins, Peter y PAIn, Rachel (2007). "Geographies of age: thinking relationally». Area, 29, (3), 287-294.

Jarrot, Shannon. E. y Weintraub, Aarón P. C. (2007). «Los centros intergeneracionales: un modelo práctico». En: SÁNCHEZ, Mariano (ed.). Las relaciones intergeneracionales. Hacia una sociedad para todas las edades. Barcelona: Estudios Sociales, (23), Obra Social, Fundación la Caixa, 141-169. <https://obrasocial.lacaixa.es/ deployedfiles/obrasocial/Estaticos/pdf/Estudios_sociales/vol23_es.pdf> [consulta: 17 de julio de 2014].

Lladó Olivera, Mónica (2010). Representaciones sociales: adultos mayores y espacios públicos en la ciudad de Montevideo. Montevideo (Uruguay): Universidad de La República, Facultad de Humanidades y Ciencias de la Educación. <http:// www.academia.edu/3176999/Representaciones_sociales_adultos_mayores_y_ espacios_p\%C3\%BAblicos_en_la_Ciudad_de_Montevideo> [consulta: 28 de octubre de 2014].

Newman, Sally y SÁnchez, Mariano (2007). «Los programas intergeneracionales: concepto, historia y modelos». En: SÁnchez, Mariano (ed.). Las relaciones intergeneracionales. Hacia una sociedad para todas las edades. Barcelona: Estudios Sociales, Obra Social, Fundación la Caixa (23) 38-69. <https://obrasocial.lacaixa.es/ deployedfiles/obrasocial/Estaticos/pdf/Estudios_sociales/vol23_es.pdf> [consulta: 27 de junio de 2014].

McCrea, James M.; Weissman, Merrille; y Thorpe-Brown, Gina (2004). Connecting the generations: A practical guide for developing intergenerational programs. Pittsburgh, PA: Generations Together.

ONU (2003). Declaración Politica y Plan de Acción Internacional de Madrid sobre el Envejecimiento. Segunda Asamblea Mundial sobre envejecimiento. Nueva York: 
Naciones Unidas. <http://undesadspd.org/Portals/0/ageing/documents/FulltextSP.pdf $>$ [consulta: 15 de julio de 2014].

Ortiz, Anna; Prats, Maria; y Baylina, Mireia (2014). «Procesos de apropiación adolescente del espacio público: otra cara de la renovación urbanística en Barcelona». Boletín de la Asociación de Geógrafos Españoles, (65), 37-57.

PAIN, Rachel (2005). Intergenerational relations and practice in the development of sustainable communities. International Centre for Regional Regeneration and Development Studies (ICRRDS) Durham University. <http:/www.centreforip.org.uk/ res/documents/publication/ODPM\%20intergenerational\%20report.pdf> [consulta: 26 de enero de 2015].

Prats, Maria; Baylina, Mireia; y Ortiz, Anna (2012a). «Métodos cualitativos y perspectivas alternativas para el estudio de los espacios públicos urbanos». En: Royé Dominic et al. (coord.). Respuestas de la Geografía Ibérica a la crisis actual. Santiago de Compostela: Universidad de Santiago de Compostela, 754-764.

Prats, Maria; Baylina, Mireia y Ortiz, Anna (2012b). «Los lugares de la amistad y la vida cotidiana de chicas y chicos adolescentes en un barrio de Barcelona». Revista latinoamericana de Geografia e Gênero, 3, (2), 116-124.

Romero, Ximena; Ruiz, Elisa Dulcey; y Brigeiro, Mauro (eds.) (2009). Hacia una sociedad para todas las edades: experiencias latinoamericanas de relaciones intergeneracionales. Santiago de Chile: Red Latinoamericana de Gerontología. <http://www. gerontologia.org/portal/archivosUpload/Libro-RLG-relaciones-intergeneracionales.pdf> [consulta: 22 de enero de 2015].

Sánchez, Mariano; Kaplan Matthew y SAÉz, Juan (2010). Los programas intergeneracionales. Guí introductoria. Madrid: Instituto de Mayores y Servicios Sociales (IMSERSO). <http://www.aepumayores.org/sites/default/files/Programas_Intergeneracionales_Coleccion_Manuales_Guias_IMSERSO_\%202010.pdf> [consulta: 7 de julio de 2014].

Schlack, Elke (2007). «Espacio Público». $A R Q$, 65, 25-27.

The Beth Johnson Foundation (2010). «Towards More Confident Communities. A Review of Community Reassurance at Neighborhood Level and Practical Guidance on Intergenerational Approaches». The Beth Johnson Foundation. <http:// www.centreforip.org.uk/res/documents/publication/Twoards\%20more\%20Confident\%20Communities.pdf> [consulta: 4 de junio de 2014].

Ventura-Merkel, Catalina y Lidoff, Lorena (1983). Community planning for intergenerational programming. Estados Unidos: Smithsonian Libraries.

VANDERBECK, Robert M. (2007). «Intergenerational geographies: age relations, segregation and re-engagements». Geography Compass, 1, 200-221. <http://dx.doi.org/10.1111/j.1749-8198.2007.00012.x>

VV. AA. (2008). Definición de La Antigua Guatemala. Documento inédito. <http:// www.riicotec.org/InterPresent2/groups/imserso/documents/binario/documento7definicindelaantigua.pdf> [consulta: 12 de enero de 2015].

VV. AA. (2009). Informe sobre la situación en siete países de Iberoamérica. Enlace, Revista de Riicotec. Monográfico, Relaciones Intergeneracionales, 1-38. <http://sid. usal.es/idocs/enlace/mri/enlacemri.pdf> [consulta: 12 de enero de 2015].

Zeldin, Shepherd; Larson, Reed; Camino, Linda y O'Connor, Cailin (2005). «Intergenerational Relationships and Partnerships in Community Programs: Purpose, Practice and Directions for research». Journal of Community Psychology, 33, (1), $1-10$. 